\title{
Experimental Evaluation of a R-EAM and Noise Impact Analysis for UWB and Wi-Fi Transmission in RoF Networks
}

\author{
L. M. Pessoa · J. M. B. Oliveira · D. V. Coelho · J. C. S. Castro · H. M. Salgado
}

Received: date / Accepted: date

\begin{abstract}
The performance of a reflective electro - absorption modulator (R-EAM) transceiver is assessed in terms of both slope efficiency (SE) and responsivity in a Radio-overFiber (RoF) network. Different biasing schemes are analyzed, specifically, zero bias (passive solution), bias for maximum SE and bias for maximum responsivity. Finally, two case studies on Multiband-OFDM Ultra-Wide-Band and Wi$\mathrm{Fi}$ are presented, for which the optimum setup parameters are determined.
\end{abstract}

Keywords Radio-over-Fiber - Electro - Absorption Modulator · Zero Bias · Ultra Wide Band

\section{Introduction}

The radio-over-fiber ( $\mathrm{RoF})$ concept involves the transmission of RF signals by an optical fiber between a control station (CS) and a number of base stations (BSs). In the base stations, the RF signal is transmitted to end users by a wireless link. Integration of both optical and wireless broadband infrastructures into the same backhaul network leads to a significant simplification and cost reduction of BSs since all routing, switching and processing are shifted to the CS. This centralization of signal processing functions enables equipment sharing, dynamic allocation of resources, and simplified system operation and maintenance. The concept of RoF is shown in Fig. 1.

RoF systems can be completely transparent to all signals

L. M. Pessoa, J. M. B. Oliveira, D. V. Coelho, J. C. S. Castro and H. M. Salgado are with INESC Porto, Porto, Portugal

L. M. Pessoa, J. M. B. Oliveira, D. V. Coelho and H. M. Salgado are also with Faculdade de Engenharia, Universidade do Porto, Porto, Portugal E-mail: \{lpessoa, jmbo, dcoelho, jcastro, hsalgado\}@inescporto.pt transmitted in the optical channel. It has been experimentally shown that RoF networks are well suited to simultaneously transport several wireless standards like wideband code division multiple access (WCDMA), IEEE 802.11 wireless local area network (WLAN) [1], global system for mobile communications (GSM) [2], WiMAX [3] and ultra-wide band (UWB) $[4,5]$. Moreover, the RoF systems are attractive for future avionics communication networks since they are lightweight and immune to electromagnetic interference. Furthermore they facilitate the provision of wireless services to passengers and satisfy the requirements for future RF communications between the aircraft and earth stations.

In a RoF system, an optically modulated mm-wave signal can suffer several impairments namely nonlinear distortion and power penalty from the E/O/E conversion process, chromatic dispersion and attenuation from the optical fiber. Moreover, optical sources with narrow line-widths are required to minimize degradation due to phase noise and, hence, very stable and expensive lasers are mandatory [6]. For the downlink signal transmission an ultra-stable and common optical source can be used. Yet, in the uplink, it is not attractive in terms of complexity, size, power consumption and cost to have an optical source for each BSs. Furthermore, by eliminating the need of an optical source at each BS, the wavelength assignment is also performed at the $\mathrm{CO}$, turning all BSs to be colorless.

In this paper we study the performance of a reflective electro - absorption modulator (R-EAM) transceiver in a RoF network. In section 2, a description of the EAM is performed and its role as a transceiver in RoF systems is discussed. In section 3 we experimentally characterize the R-EAM in terms of Slope Efficiency (SE) and responsivity $\left(R_{e}\right)$ for different wavelengths, optical powers and bias points. In sec- 


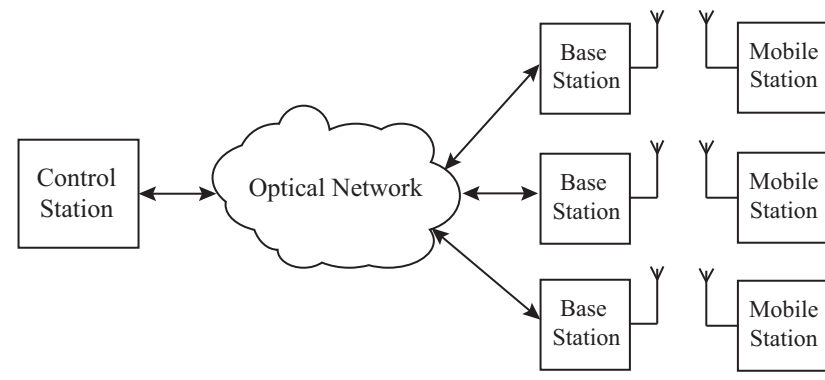

Fig. 1 Radio-over-fiber system concept

tion 4, a case study of UWB signal transmission is addressed, where the optimum operation points are discussed for different scenarios: bias for maximum $\mathrm{SE}$, bias for maximum $R_{e}$ and zero bias. Finally, conclusions are given in section 5 .

\section{Electro - Absorption Transceivers in RoF Systems}

There are two main BS schemes that are usually used in source free RoF systems. The first scheme is based on an external modulator, photoreceiver and optical filtering techniques that use wavelength reuse or a more convenient method in which the optical carrier is provided remotely from the CO [7-9]. Another scheme of source free BSs is based on a single electro - absorption waveguide device in which a single component acts as a modulator for the uplink and as photoreceiver for the downlink [10,11]. Therefore, this transceiver device is a very attractive solution for a full-duplex RoF transmission. Although a RoF system based on electro-absorption transceiver (EAT) leads to low power consumption as well as low component count, a more desirable solution based on powerless passive EATs have also been already reported [12]. Moreover, a dual lightwave approach can also be performed. By using two different wavelengths it is possible to optimize the insertion loss of the transceiver for both uplink and downlink signal transmission, i.e., segregation of uplink and downlink signals is performed using different wavelengths. Some authors have been appointing optical transceivers as a key component to realize low-cost BS in the long run [12]. The reflective EAM is an interesting device for operation simultaneously as a modulator and photoreceiver where the rear facet is coated with high reflection layer. The performance of the R-EAM will be discussed in the next section.

\section{R-EAM performance analysis}

The experimental setup for the characterization of the $60 \mathrm{GHz}$ R-EAM transceiver (CIP EAM-R-60-C-V-FCA) is shown in Fig. 2. Both electrical and optical test signals are used in or-

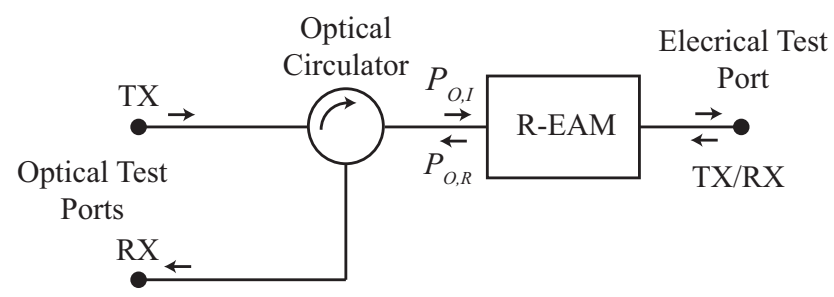

Fig. 2 R-EAM characterization setup
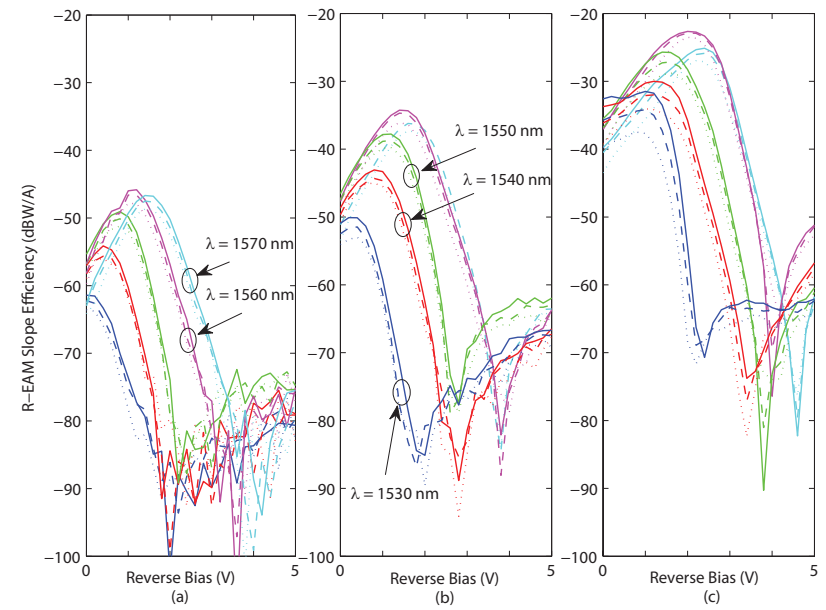

Fig. 3 EO response versus reverse bias voltage, for different wavelengths and frequencies and $P_{O, I}$, (a) $-6 \mathrm{dBm}$, (b) $0 \mathrm{dBm},(\mathrm{c})+7 \mathrm{dBm}$. Solid, dashed and dotted lines correspond to frequencies of 2.4, 5, 15 $\mathrm{GHz}$, respectively

der to obtain the electro-optical (EO) and optical to electrical (OE) response, which correspond to the slope efficiency and responsivity, respectively. We assume the decibel units for these variables to be obtained by $20 \log _{10}(\cdot)$, as defined in the measurements of the laboratory equipment. Fig. 3 shows the EO response as a function of reverse bias voltage, for different frequencies and wavelengths. The R-EAM average optical input power, $P_{O, I}$, is varied by controlling the CW laser output power. It is apparent that the optimum bias voltage increases with both wavelength and the optical input power. It is also apparent that SE degrades slightly with frequency especially for high $P_{O, I}$, and lower wavelengths. Furthermore, it is easily seen that $\mathrm{SE}$ at the optimum bias voltage increases with $P_{O, I}$. Fig. 4 shows the OE response as a function of reverse bias voltage, for different frequencies and wavelengths. Due to the similarity of results, only two wavelengths are plotted, in order to allow a clear visualization. Similarly to what was observed for the EO case, the responsivity degrades with frequency, particularly for high values of $P_{O, I}$, shorter wavelengths and decreasing reverse bias. Nevertheless, responsivity is shown to be more affected than SE. Furthermore, results also show that responsivity increases monotonically with reverse bias voltage. 


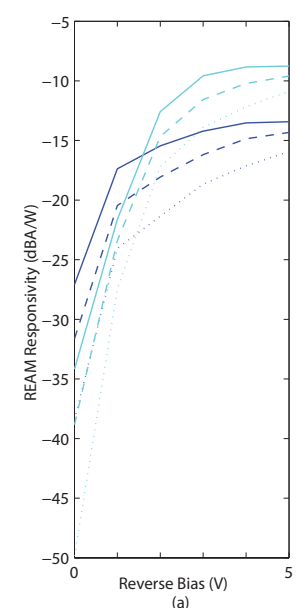

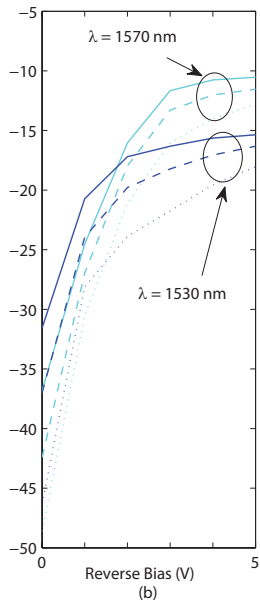

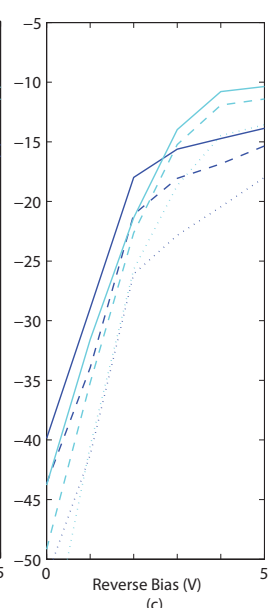

Fig. 4 OE response versus reverse bias voltage, for different wavelengths and frequencies and $P_{O, I}$, (a) $-6 \mathrm{dBm}$, (b) $0 \mathrm{dBm}$, (c) $+7 \mathrm{dBm}$. Solid, dashed and dotted lines correspond to frequencies of 2.4, 5, 15 $\mathrm{GHz}$, respectively

The optimum bias points for maximum slope efficiency and responsivity have been extracted from the results of Fig. 3 and 4. Considering these values, the R-EAM performance is assessed in terms of slope efficiency and responsivity for the following cases: bias for maximum SE, bias for maximum $R_{e}$ and zero bias. The results of this analysis are plotted in Fig. 5 and 6. The results in Fig. 5 show that the best performance is obtained for a wavelength of $1560 \mathrm{~nm}$, when the EAM is biased for maximum SE. However, when zero biased, the optimum wavelength is reduced to $1530 \mathrm{~nm}$ for $P_{0, I}=7 \mathrm{dBm}$, where a penalty of $13 \mathrm{~dB}$ is incurred, compared to the case of maximum SE. Finally, when the EAM is biased for maximum responsivity, the optimum wavelength is $1560 \mathrm{~nm}$ and the $\mathrm{SE}$ decreases by $15 \mathrm{~dB}$, compared to the zero bias case. It has also been verified experimentally that, as expected [9], the slope efficiency is proportional to the input optical power, as seen in Fig. 5 (a) and (b), until it saturates at high optical powers, as shown by Fig. 5 (c). Nevertheless, high optical input powers should be used in order to maximize the SE. Concerning the EAM responsivity, its value is optimum for $P_{O, I}=-6 \mathrm{dBm}$, while a noticeable reduction is observed with increasing $P_{O, I}$, especially for zero bias. In both cases of biasing for maximum $R_{e}$ and $\mathrm{SE}$, the EAM responsivity improves with increasing wavelength, except for high $P_{O, I}=+7 \mathrm{dBm}$, where its maximum is achieved at $\lambda=1560 \mathrm{~nm}$. The best responsivity values for both cases of biasing for maximum $R_{e}$ and SE are $R_{e}=\{-9.6,-18.6\} \mathrm{dBA} / \mathrm{W}$, respectively. For the zero bias case, the responsivity decreases with wavelength for both $P_{O, I}=\{-6,0\} \mathrm{dBm}$, reaching a maximum of $-31 \mathrm{dBA} / \mathrm{W}$ for $P_{O, I}=-6 \mathrm{dBm}$ and $1530 \mathrm{~nm}$. However, for $P_{O, I}=+7 \mathrm{dBm}$ the responsivity is relatively constant with the wavelength, except for $1570 \mathrm{~nm}$ where it degrades by $6 \mathrm{~dB}$. The re-

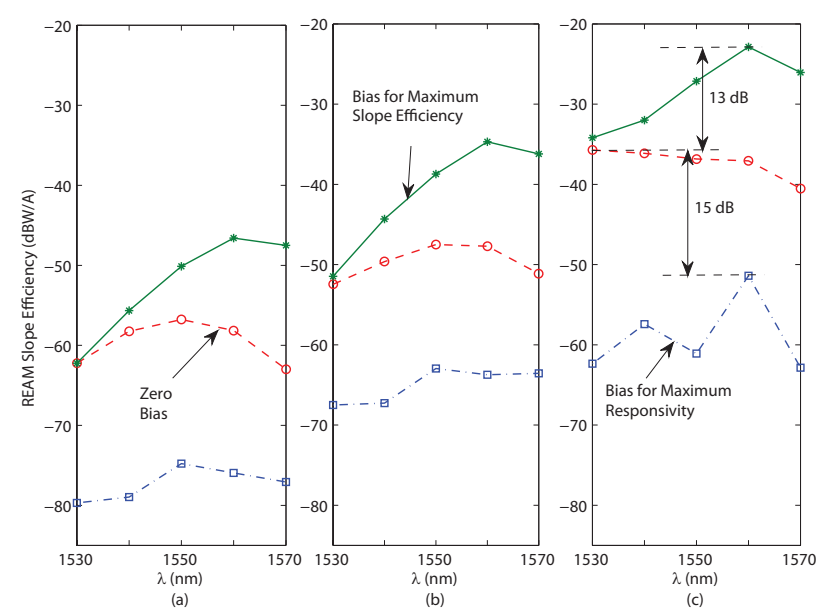

Fig. 5 EO response versus wavelength, for different bias configurations and $P_{O, I}$, (a) $-6 \mathrm{dBm}$, (b) $0 \mathrm{dBm}$, (c) $+7 \mathrm{dBm}$.
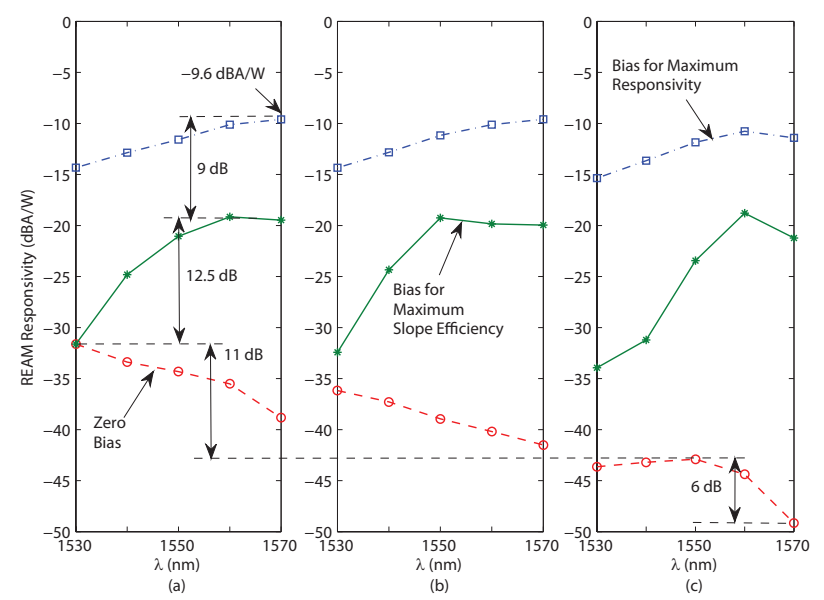

Fig. 6 OE response versus wavelength, for different bias configurations and $P_{O, I}$, (a) $-6 \mathrm{dBm}$, (b) $0 \mathrm{dBm},(\mathrm{c})+7 \mathrm{dBm}$.

sponsivity degradation with increasing $P_{O, I}$, translates into a penalty of $11 \mathrm{~dB}$ when $P_{O, I}$ is increased from -6 to $+7 \mathrm{dBm}$. The R-EAM performance was also assessed in terms of reflected optical power, $P_{O, R}$, for different values of input optical power $P_{O, I}$, the results of this analysis being shown in Fig. 7. The reflected optical power is a relevant parameter with impact in the optical to electrical conversion. Therefore, this analysis considered the wavelengths of $1550 \mathrm{~nm}$ and $1560 \mathrm{~nm}$, because these provide the best performance according to the measurements of slope efficiency given in Fig. 6. The results given by the current analysis, indicate that the reflected optical power tends to decrease with increasing reverse bias voltage. Furthermore, the optical power reflected at the wavelength of $1550 \mathrm{~nm}$ is generally lower than that reflected at $1560 \mathrm{~nm}$, except for higher reverse bias at low optical input powers. 


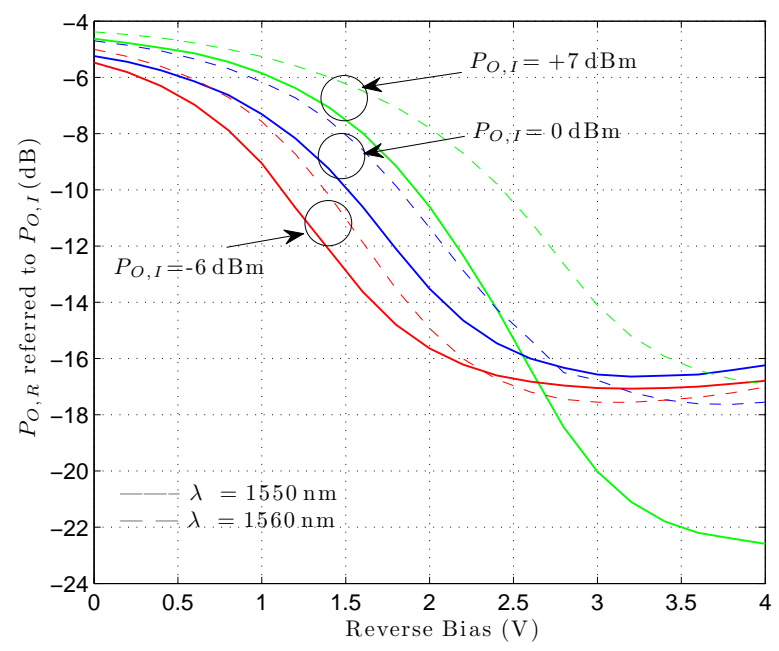

Fig. 7 R-EAM reflected optical power, $P_{O, R}$ referred to the input optical power $P_{O, I}$

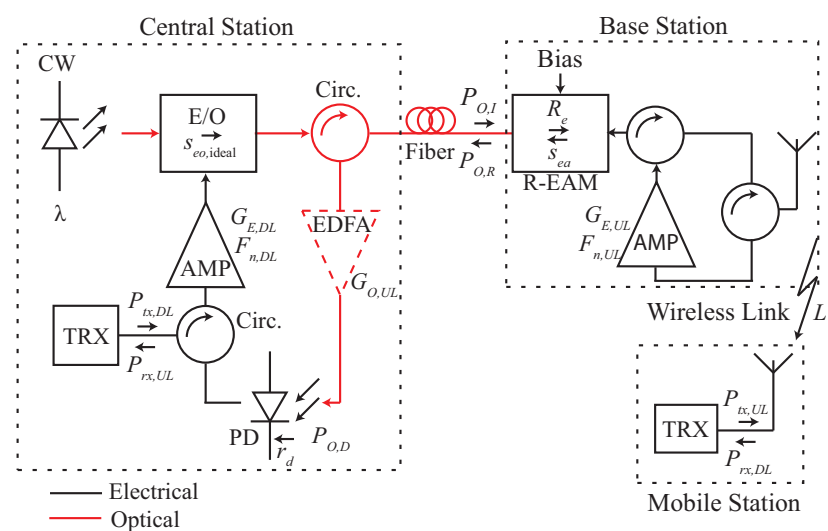

Fig. 8 Considered setup for performance assessment. CW represents a continuous wave light source. Both TRX shown, represent the transceivers at the Control Station and Mobile Station. PD represents the central station photodiode. The EDFA depicted, represents an optional Erbium Doped Fiber Amplifier.

\section{System performance assessment}

In this section, we consider the application of the R-EAM as a base station in a bidirectional transmission system, typical in a RoF network. A diagram of the application scenario is shown in Fig. 8. The RF downlink (DL) signal, generated by the central station transceiver (TRX) with a power of $P_{t x, D L}$, passes through an electrical circulator and amplifier with gain $G_{E, D L}$, and drives an E/O modulator, considered ideal in the present analysis. The optical downlink signal then passes through an optical circulator, and reaches the R-EAM through an optical fiber, with an incident optical power of $P_{O, I}$. The RF modulated optical signal is converted to the electrical domain by the R-EAM with a responsivity $\left(R_{e}\right)$, and then reaches the mobile station through the wireless channel, which induces a signal loss of $L$. Note that the attenuation parameter $L$ already includes both BS and
MS antenna gains. Conversely, the RF uplink (UL) signal is generated by the mobile station transceiver with a power of $P_{t x, U L}$, and reaches the base station after being attenuated by the wireless link. The weak RF uplink signal can be amplified electrically $\left(G_{E, U L}\right)$ before being converted from the electrical to the optical domain with a conversion efficiency given by the EAM slope efficiency, $s_{e a}$, modulating the reflected optical carrier of power $P_{O, R}$. The uplink signal might be further optically $\left(G_{O, U L}\right)$ amplified before reaching the central station transceiver. This optical amplification is only adequate if the optical power is low (typically less than $-3 \mathrm{dBm}$ ), so that the noise added by the EDFA is still below the noise level at the receiver [13]. In the following analysis, we assume $P_{O, R} \cong P_{O, D}$, since the optical circulator loss and the use of an EDFA are not considered. The metric used to evaluate performance of the system is the signal to noise ratio (SNR).

The SNR of the RF signal arriving at the mobile station can be written as:

$\mathrm{SNR}_{r x, M S}=\frac{\left\langle I_{r x, D L}^{2}\right\rangle}{\sigma_{n, M S}^{2}}=\frac{\left\langle I_{t x, D L}^{2}\right\rangle G_{D L}}{\left\langle I_{t, M S}^{2}\right\rangle}$

where,

$$
\begin{aligned}
G_{D L}= & G_{E, D L} s_{e o, \text { ideal }}^{2} R_{e}^{2} L \\
\left\langle I_{t, M S}^{2}\right\rangle= & \left(4 T+T_{a}\right) k \Delta_{f} / R_{M S} \\
& +\left(F_{n, D L}-1\right) k T G_{D L} \Delta_{f} / R_{E O} \\
P_{t x, D L}= & R_{C S}\left\langle I_{t x, D L}^{2}\right\rangle
\end{aligned}
$$

Therefore, the SNR at the receiver can be written as the following ratio:

$\mathrm{SNR}_{r x, M S}=\frac{\left(P_{t x, D L} / \Delta_{f}\right) G_{D L} / R_{E O}}{\left(4 T+T_{a}\right) k / R_{M S}+\left(F_{n, D L}-1\right) k T G_{D L} / R_{E O}}$

where $R_{M S}$ represents the impedance of the mobile station circuitry, $R_{E O}$ the impedance of the E/O converter circuitry, $\Delta_{f}$ the transmission bandwidth, $s_{e o \text {,ideal }}$ the slope efficiency of the ideal E/O modulator, $F_{n, D L}$ the noise factor of the electrical amplifier that precedes the modulator, $k$ is Boltzmann's constant, $T=290 \mathrm{~K}$ and $T_{a}=120 \mathrm{~K}$ is the antenna temperature. Since the link gain $\left(G_{D L}\right)$ is low, the noise at the receiver is dominated by the thermal noise of the receiver circuitry itself. Furthermore, since the R-EAM efficiency is higher in the downlink, regardless of the bias conditions, the SNR is considerably higher than in the uplink.

The SNR of the RF signal arriving at the central station can be written as:

$\mathrm{SNR}_{r x, C S}=\frac{\left\langle I_{r x, U L}^{2}\right\rangle}{\sigma_{n, C S}^{2}}=\frac{\left\langle I_{t x, U L}^{2}\right\rangle G_{U L}}{\left\langle I_{r i n}^{2}\right\rangle+\left\langle I_{s n}^{2}\right\rangle+\left\langle I_{t, C S}^{2}\right\rangle}$

where,

$G_{U L}=G_{E, U L} s_{e a}^{2} r_{d}^{2} L$ 


$$
\begin{aligned}
\left\langle I_{\text {rin }}^{2}\right\rangle= & r_{d}^{2}\left\langle P_{O, D}^{2}\right\rangle 10^{\mathrm{RIN} / 10} \Delta_{f} \\
\left\langle I_{s n}^{2}\right\rangle= & 2 q r_{d}\left\langle P_{O, D}\right\rangle \Delta_{f} \\
\left\langle I_{t, C S}^{2}\right\rangle= & 4 k T \Delta_{f} / R_{\mathrm{L}} \\
& +\left(T_{a}+\left(F_{n, U L}-1\right) T\right) k G_{E, U L} s_{e a}^{2} r_{d}^{2} \Delta_{f} / R_{E A M} \\
P_{t x, U L}= & R_{M S}\left\langle I_{t x, U L}^{2}\right\rangle
\end{aligned}
$$

where $R_{E A M}$ represents the impedance of the R-EAM circuitry.

The noise terms are referred to the photodiode output, and stem from 3 main components: the relative intensity noise (RIN) from the laser source (proportional to the square of $\left\langle P_{O, D}\right\rangle$ ), the shot noise (proportional to $\left\langle P_{O, D}\right\rangle$ ), and the last term is the thermal noise from both the photodiode load $\left(R_{\mathrm{L}}\right)$, the antenna and the uplink electrical amplifier, where $F_{n, U L}$ is the noise factor of the amplifier at the base-station. The thermal noise from the electrical transmitter at the mobile station can be neglected, due to the low total gain of the link $\left(G_{U L}\right)$. The optical power detected by the photodiode, $P_{O, D}$, is expected to have a significant impact on the noise contribution at the receiver. In the presented results, the wireless channel attenuation $(L)$ was not considered, the fiber is considered ideal, $G_{E, U L / D L}=1, F_{n, U L / D L}=1, R_{\mathrm{L}}=$ $1000 \Omega$ and $R_{M S / E O / E A M}=50 \Omega$.

\subsection{Case study: UWB MB-OFDM [ECMA-368]}

Ultra Wide Band (UWB) signals [14] are characterized by their huge bandwidth occupation, data rates up to $480 \mathrm{Mbit} / \mathrm{s}$, and very low power density $(-41.3 \mathrm{dBm} / \mathrm{MHz})$, which gives them a noise-like signal characteristic and provides both interference mitigation and very low device power consumption. The available spectrum $(3.1-10.6 \mathrm{GHz})$ is divided into 14 sub-bands, each with $528 \mathrm{MHz}$ bandwidth. The standard also defines that these bands are grouped into four groups of three bands, allowing for frequency hopping and multiple user communication in a pico-cell network [15]. Additionally, its coverage is limited to a few meters, due to the low signal intensity, making it a good candidate for deployment in future aircraft in-flight entertainment networks, where the high user density benefits from a reduction of the sharing factor between wireless cells. The present analysis considers the usage of typical UWB transceivers (Wisair DVK9110), which operate in band group 1 (from $3.168 \mathrm{GHz}$ to $4.752 \mathrm{GHz}$ ) and have a maximum transmission power of approximately $P_{t x, U L} / \Delta_{f}=-45.3 \mathrm{dBm}[16]$, when the MS antenna gain (considered $\sim 4 \mathrm{~dB}$ ) is lumped into the wireless channel attenuation. The UWB receiver sensitivity of $-70.4 \mathrm{dBm}$ at $480 \mathrm{Mbit} / \mathrm{s}$ specified in the standard [14], is not valid for an optical front-end. This value assumes a typical value of noise level in a wireless receiver of $-80.5 \mathrm{dBm}$, which indicates that a receiver should be able to meet the target performance specified in the standard, requiring no more than $\sim 10 \mathrm{~dB}$ of SNR. In the present analysis, a minimum SNR of $7.5 \mathrm{~dB}$ is considered, which has been experimentally found for the mentioned commercial UWB transceiver. The results in Fig. 9 show the SNR as a function of the reverse bias, for three different values of $P_{O, I}$, (a) $-6 \mathrm{dBm}$, (b) $0 \mathrm{dBm}$ and (c) $+7 \mathrm{dBm}$, considering a responsivity of $1 \mathrm{~A} / \mathrm{W}$ and two different wavelengths, $1550 \mathrm{~nm}$ and $1560 \mathrm{~nm}$ and RIN values, $-150 \mathrm{~dB} / \mathrm{Hz}$ and $-160 \mathrm{~dB} / \mathrm{Hz}$. Moreover, SNR results considering only the thermal noise term are also depicted for comparisson purposes. Two additional equivalent SNR limits that consider a wireless link length of $0.5 \mathrm{~m}$ and also $1 \mathrm{~m}$ are also shown in the results, which account for a signal loss of 28.5 and $34 \mathrm{~dB}$ at $4 \mathrm{GHz}$, respectively, when considering a sum antenna gain of $10 \mathrm{dBi}$, among MS and BS. Results show that for low reverse bias the impact of both shot noise and RIN increase with the incident optical power. This result comes in line with the ones of the reflected optical power given by Fig. 7. Moreover, it is clearly seen that the SNR is optimum for a wavelength of $1560 \mathrm{~nm}$ with a maximum SNR $5 \mathrm{~dB}$ better than that at $1550 \mathrm{~nm}$, although slightly worse for zero bias.

In Fig. 10 the SNR is obtained as a function of $P_{O, I}$, for the following cases: (a) zero bias, (b) optimum bias for maximum SE and (c) optimum bias for maximum SNR, considering only the wavelength of $1560 \mathrm{~nm}$. Again, two additional equivalent SNR limits that consider a wireless link length of $0.5 \mathrm{~m}$ and also $1 \mathrm{~m}$ are also shown in the results. The results considering optimum bias for maximum Slope Efficiency are, at the maximum points, $\sim 5-6 \mathrm{~dB}$ worse than those for maximum SNR, essentially because there is a compromise between signal power, which is affected by the $s_{e, a}$ as a function of bias voltage, and noise power, affected by the $P_{O, D}$ which also depends on the bias voltage.

From an overview perspective, it can be noted that the SNR performance increases with $P_{O, I}$ for a RIN of $-160 \mathrm{~dB} / \mathrm{Hz}$, whereas for $-150 \mathrm{~dB} / \mathrm{Hz}$ there is an optimum value of $P_{O, I}$ for maximum SNR. At zero bias, a maximum margin of $9 \mathrm{~dB}$ compared to the UWB SNR limit is obtained with a laser RIN of $-150 \mathrm{~dB} / \mathrm{Hz}$ for a $P_{O, I}$ of $\sim 0 \mathrm{dBm}$, whereas for a RIN of $-160 \mathrm{~dB} / \mathrm{Hz}$ the performance becomes limited by shot noise, allowing for a SNR margin of $\sim 13.5 \mathrm{~dB}$. However, the results indicate that none of such limits are achieved at zero bias, because of both RIN and shot noise limitations, which means that a totally passive base-station is not practicable, for reasonable wireless link distances. Results for optimum bias for maximum SE indicate that the SNR for a RIN of $-150 \mathrm{~dB} / \mathrm{Hz}$ practically achieves the UWB SNR limit for a wireless link distance of 0.5 meters with a $P_{O, I}$ of $\sim 6 \mathrm{dBm}$, whereas for a RIN of $-160 \mathrm{~dB} / \mathrm{Hz}$ a 1 meter link is almost achieved. Note that while the former 

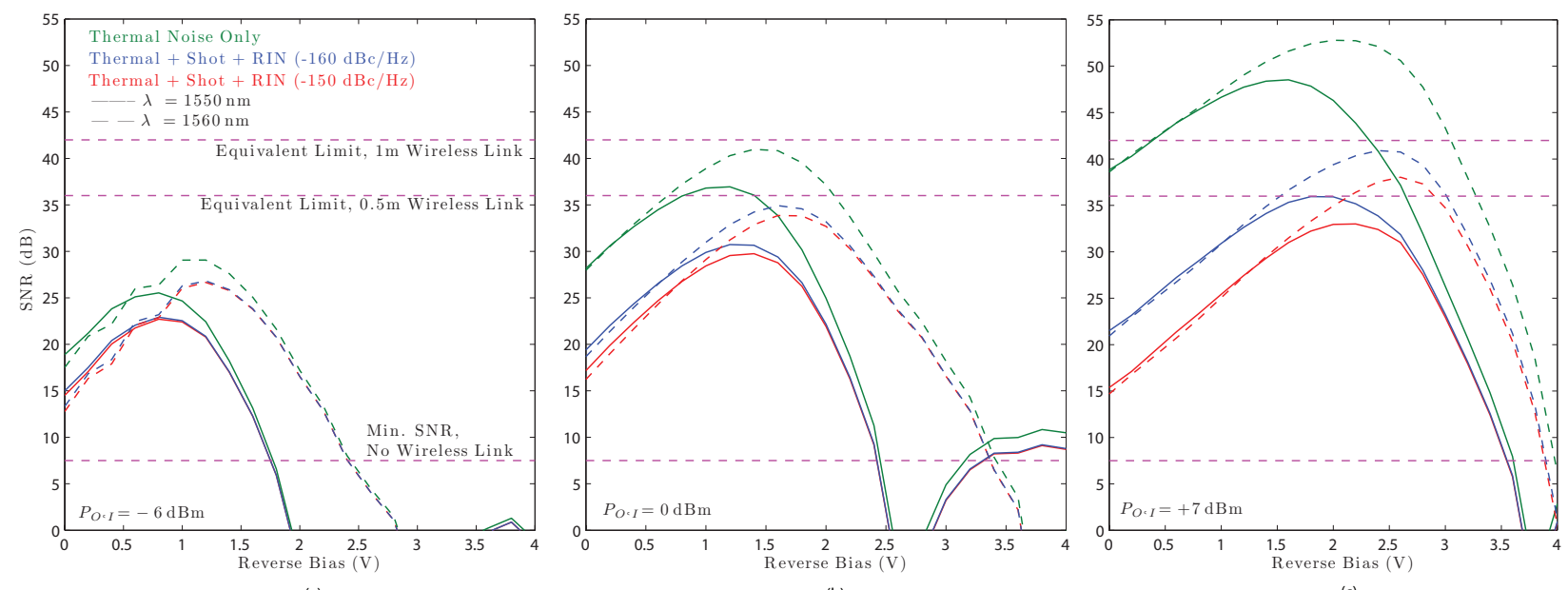

(a)

Fig. $9 \mathrm{SNR}$ as a function of reverse bias, for three different values of $P_{O, I}$, (a) $-6 \mathrm{dBm}$, (b) $0 \mathrm{dBm},(\mathrm{c})+7 \mathrm{dBm}$.

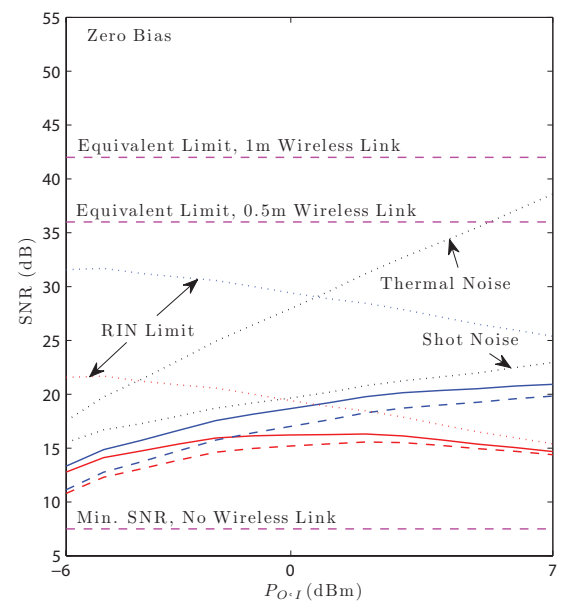

(a)

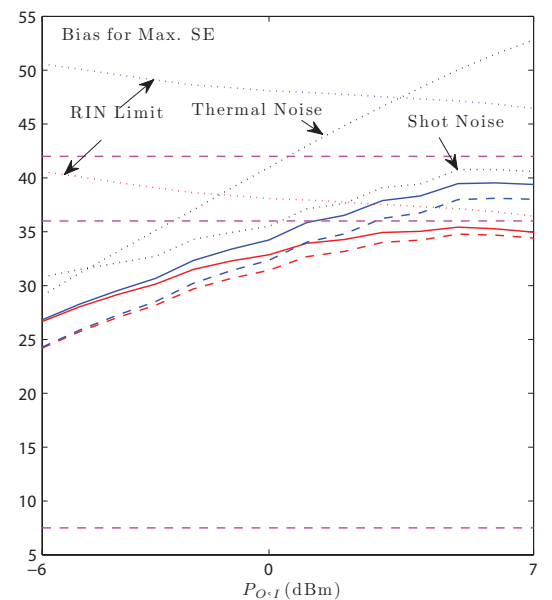

(b)

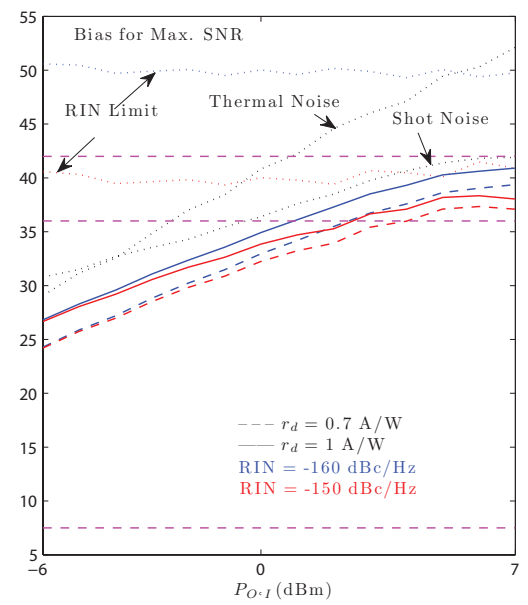

(c)

Fig. 10 SNR as a function of $P_{O, I}$, for the cases of (a) zero bias, (b) optimum bias for maximum SE and (c) optimum bias for maximum SNR.

SNR is limited by the RIN term, the latter is limited by the shot noise. At optimum bias for maximum SNR, a margin of $33.5 \mathrm{~dB}$ is obtained at the maximum $P_{O, I}$, for a RIN of $-160 \mathrm{~dB} / \mathrm{Hz}$ and $r_{d}=1 \mathrm{~A} / \mathrm{W}$, allowing for an acceptable wireless link distance of approximately 1 meter. For a RIN of $-150 \mathrm{~dB} / \mathrm{Hz}$, there is enough SNR margin to allow for a wireless link distance between 0.5 and 1 meter. Furthermore, by reducing the UWB throughput, a maximum distance of 2.8 meters would be achievable at $53.3 \mathrm{Mbit} / \mathrm{s}$, at a minimum SNR of $0 \mathrm{~dB}$. Since the R-EAM slope efficiency at optimum bias for maximum responsivity is worse than that at zero bias, there is no advantage in analyzing that scenario.

\subsection{Case study: MIMO-OFDM Wireless LAN [IEEE802.11n]}

Multiple-Input Multiple Output (MIMO)-OFDM technology is used for the wireless LAN systems (IEEE802.11n [17]), typically referred as WiFi. The WiFi receiver sensitivity of $-61 \mathrm{dBm}$ at $300 \mathrm{Mbit} / \mathrm{s}$ specified in the standard [17], is not valid for an optical front-end. Instead, a minimum SNR of $20 \mathrm{~dB}$ can be specified, considering the typical noise level of a WiFi receiver at $-94 \mathrm{dBm}$ in a $40 \mathrm{MHz}$ bandwidth, and a typical sensitivity from a commercial IEEE802.11n of $-74 \mathrm{dBm}$, in the $2.4 \mathrm{GHz}$ band [18].

The results depicted in Fig. 11 and 12 are analogous to those shown in Fig. 9 and 10 for the UWB case study. The results were updated to reflect a typical transmitted power of $16 \mathrm{dBm}$ in a $40 \mathrm{MHz}$ bandwidth [18], equivalent to $0 \mathrm{dBm} / \mathrm{MHz}$ power spectral density, which is $\sim 45.3 \mathrm{~dB}$ higher than that of a UWB transceiver. The results show three different equivalent SNR limits that consider wireless link lengths of $\{1,5$, $20\} \mathrm{m}$, which account for a signal loss of $\{30,44,56\} \mathrm{dB}$ at $2.4 \mathrm{GHz}$, respectively, when considering a sum antenna gain of $10 \mathrm{dBi}$, among MS and BS. Given that the transmitted power is much higher than that of the UWB, the expected 

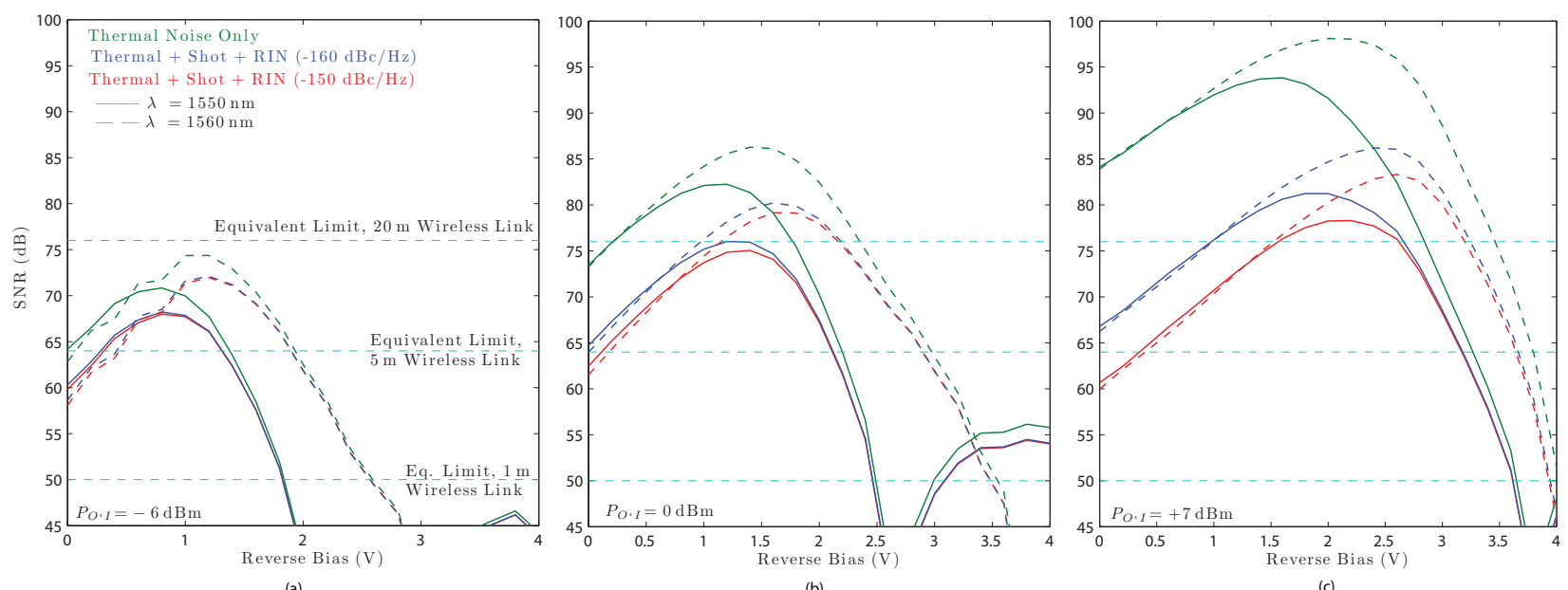

Fig. $11 \mathrm{SNR}$ as a function of reverse bias, for three different values of $P_{O, I},($ a) $-6 \mathrm{dBm}$, (b) $0 \mathrm{dBm}$, (c) $+7 \mathrm{dBm}$.
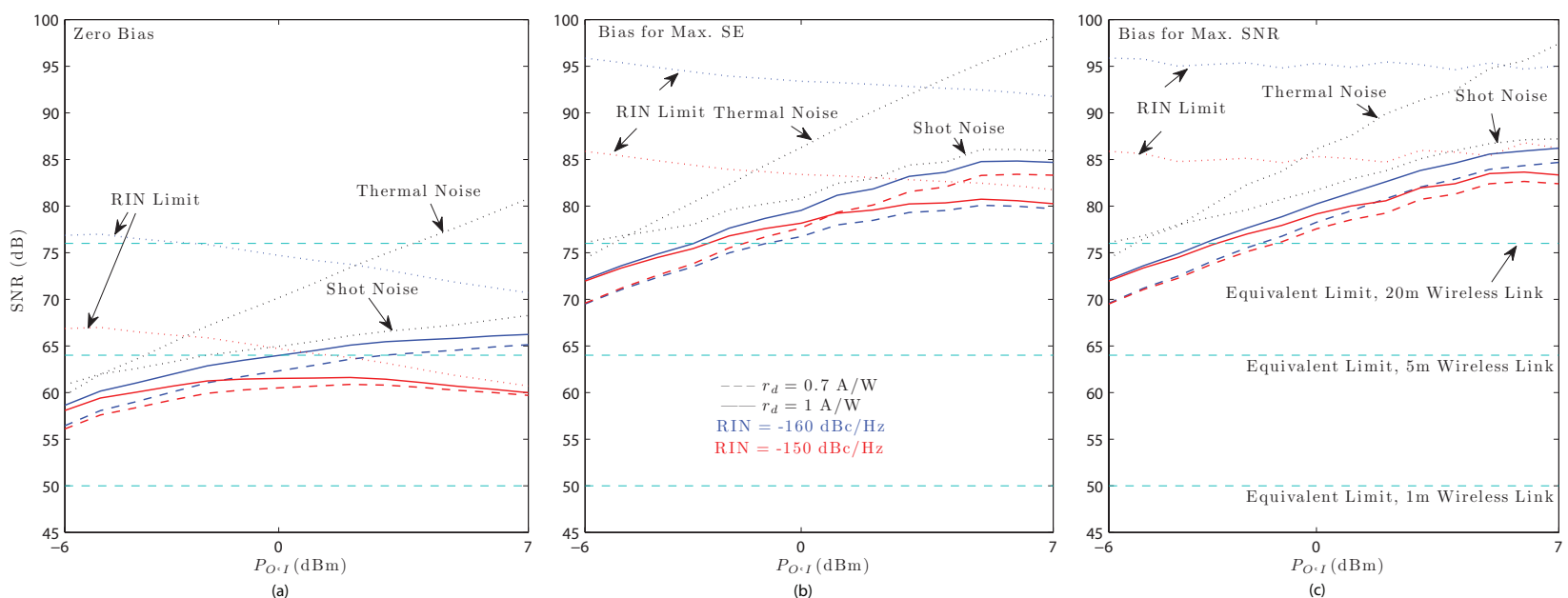

Fig. 12 SNR as a function of $P_{O, I}$, for the cases of (a) zero bias, (b) optimum bias for maximum SE and (c) optimum bias for maximum SNR.

tolerable wireless link distances are much higher, in spite of the higher SNR requirement. Specifically, the zero bias configuration is suitable for a $5 \mathrm{~m}$ wireless link, provided that a laser RIN of $-160 \mathrm{~dB} / \mathrm{Hz}$ is used. If a laser RIN value of $-150 \mathrm{~dB} / \mathrm{Hz}$ is used, an approximate wireless link of $\sim 4 \mathrm{~m}$ is acceptable. Furthermore, more than $20 \mathrm{~m}$ wireless link is possible when the EAM is biased for maximum SNR.

Similarly to the UWB signal transmission case, the maximum SNR for a RIN of $-160 \mathrm{~dB} / \mathrm{Hz}$ for the three bias points considered is mainly limited by the shot noise whereas the maximum SNR for a RIN of $-150 \mathrm{~dB} / \mathrm{Hz}$ is limited by the RIN term. Nevertheless, considerable wireless link distances are achieved, even for zero bias.

\section{Conclusion}

This paper presented an analysis on the performance of the slope efficiency and responsivity of a R-EAM transceiver for different wavelengths, optical powers and bias points. Furthermore, its performance was evaluated for the case studies of Ultra Wide Band (ECMA-368) and Wireless LAN (IEEE802.11n) standards. The optimum operation point was found to be the biasing for maximum SNR, using an high optical input power, and a wavelength of $1560 \mathrm{~nm}$. Although a zero bias configuration is an attractive technique, it is not suitable to provide a reasonable wireless link distance for UWB, while being suitable to enable a $\sim 4-5 \mathrm{~m}$ wireless link for WiFi. We conclude that the measured R-EAM is a suitable option to be implemented as a base-station in future aircraft infotainment networks. One of the performancelimiting factors comes from the laser RIN that imposes a barrier to the achievable SNR, especially for a zero biased modulator. A laser RIN of $-160 \mathrm{~dB} / \mathrm{Hz}$ would be required in order to avoid the RIN limitation. Additionally, in this case the performance becomes limited by shot noise. Although the zero bias case seems limitative, the R-EAM can be optimum biased using a small battery, which can last for several 
months, allowing the base-station to operate as a passive device.

Acknowledgements We acknowledge funding from FCT and program POCTI / FEDER under the National Plan for Scientific Hardware Renewal with grant REEQ / 1272/EEI/2005.

This work was supported in part by FCT under the project "Design and Optimisation of WDM Millimetre-Wave Fibre- Radio Systems" (PTDC / EEA - TEL/68974/2006) and EC Framework 7 (FP7) project DAPHNE (www.fp7daphne.eu) "Developing aircraft photonic networks" (grant ACP8-GA - 2009-233709).

D. V. Coelho also acknowledges support from FCT through a PhD grant.

\section{References}

1. J. Mitchell, "Performance of OFDM at $5.8 \mathrm{GHz}$ using radio over fibre link," IEEE Electronics Letters, vol. 40, no. 21, pp. 13531354, (2004).

2. P. K. Tang, L. C. Ong, A. Alphones, B. Luo, and M. Fujise, "PER and EVM measurements of a radio-over-fiber network for cellular and WLAN system applications," IEEE Journal of Lightwave Technology, vol. 22, no. 11, pp. 2370 - 2376, (2004).

3. N. Gomes, M. Morant, A. Alphones, B. Cabon, J. Mitchell, C. Lethien, M. Csörnyei, A. Stöhr, and S. Iezekiel, "Radio-overfiber transport for the support of wireless broadband services [invited]," Journal of Optical Networking, vol. 8, no. 2, pp. 156-178, (2009).

4. M. Jazayerifar, B. Cabon, and J. Salehi, "Transmission of multiband OFDM and impulse radio ultra-wideband signals over single mode fiber," IEEE Journal of Lightwave Technology, vol. 26, no. 15 , pp. 2594-2603, (2008).

5. M. Sauer, A. Kobyakov, and J. George, "Radio over fiber for picocellular network architectures," IEEE Journal of Lightwave Technology, vol. 25, no. 11, pp. 3301-3320, (2007)

6. C. Lim, A. Nirmalathas, M. Bakaul, P. Gamage, K. Lee, Y. Yang, D. Novak, and R. Waterhouse, "Fiber-wireless networks and subsystem technologies," IEEE Journal of Lightwave Technology, vol. 28 , no. 4, pp. 390-405, (2010).
7. Z. Jia, J. Yu, and G. Chang, "A full-duplex radio-over-fiber system based on optical carrier suppression and reuse," IEEE Photonics Technology Letters, vol. 18, no. 16, pp. 1726-1728, (2006).

8. L. Chen, H. Wen, and S. Wen, "A radio-over-fiber system with a novel scheme for millimeter-wave generation and wavelength reuse for up-link connection," Photonics Technology Letters, IEEE, vol. 18, no. 19, pp. 2056-2058, (2006).

9. C. Cox, Analog optical links: theory and practice. Cambridge Univ Pr, (2004).

10. D. Wake, D. Johansson, and D. Moodie, "Passive picocell: a new concept in wireless network infrastructure," IEEE Electronics Letters, vol. 33, no. 5, pp. 404-406, (1997).

11. A. Stohr, K. Kitayama, and D. Jager, "Full-duplex fiberoptic RF subcarrier transmission using a dual-function modulator/photodetector," IEEE Transactions on Microwave Theory and Techniques, vol. 47, no. 7, pp. 1338-1341, (1999).

12. K. Kitayama, "Architectural considerations of fiber-radio millimeter-wave wireless access systems," Fiber and Integrated Optics, vol. 19, no. 2, pp. 167-186, (2000).

13. G. Keiser, Optical fiber communications. Wiley Online Library, (2000).

14. H. PHY and M. Standard, "ECMA international ECMA-368," (2005).

15. Y. Chen, J. Zhang, and A. Jayalath, "Multiband-ofdm uwb vs ieee802. 11n: System level design considerations," in IEEE Ve hicular Technology Conference, vol. 4, pp. 1972-1976, (2006).

16. J. Oliveira, S. Silva, L. Pessoa, D. Coelho, H. Salgado, and J. Castro, "UWB radio over perfluorinated GI-POF for low-cost inbuilding networks," in IEEE Topical Meeting on Microwave Photonics, pp. 317-320, (2010).

17. IEEE, "IEEE Standard for Information technologyTelecommunications and information exchange between systems-Local and metropolitan area networks-Specific requirements Part 11: Wireless LAN Medium Access Control (MAC) and Physical Layer (PHY) Specifications Amendment 5: Enhancements for Higher Throughput," IEEE Std 802.11n-2009, pp. c1 -502, 29 (2009).

18. W. N. Corporation, "Product Specifications of DNMA-92, An IEEE $802.11 \mathrm{n} \mathrm{a/b/g/n} \mathrm{Mini-PCI} \mathrm{module,} \mathrm{version} \mathrm{1.6,"} \mathrm{April}$ (2009). 